\title{
STRATEGIES AND TOOLS FOR PREVENTING NEUROTOXICITY: TO TEST, TO PREDICT AND HOW TO DO IT.
}

Jordi Llorens ${ }^{a b,}$, , Abby Li ${ }^{i}$, Sandra Ceccatelli ${ }^{d}$, Cristina Suñol ${ }^{e}$

a Department of Physiological Sciences II, Universitat de Barcelona, 08907 Hospitalet de Llobregat, Spain

b Institut d'Investigació Biomèdica de Bellvitge, IDIBELL, Hospitalet de Llobregat, Spain ${ }^{c}$ c/o Rebecca Edwards, Exponent Health Sciences, 149 Commonwealth Drive, San FranciscoMenlo Park CA 94025, USA. e-mail: abbyli@exponent.com

${ }^{d}$ Department of Neuroscience, Karolinska Institutet, 17177 Stockholm, Sweden. email: sandra.ceccatelli@ki.se

e Institut d'Investigacions Biomèdiques de Barcelona (IIBB-CSIC-IDIBAPS;

CIBERESP), ), c/ Rosselló 161, 6ª . Room 629, 08036 Barcelona, Spain. e-mail: csenqi@iibb.csic.es

"Corresponding author at Department of Physiological Sciences II, Universitat de Barcelona, Feixa Llarga s/n, 08907 Hospitalet de Llobregat, Spain. Tel.: 3493322 4087. E-mail: illorens@ub.edu. 


\section{ABSTRACT}

A change in paradigm is needed in the prevention of toxic effects on the nervous system, moving from its the present reliance solely on data from animal testing to a prediction model mostly based on in vitro toxicity testing and in silico modelling. According to the report published by the National Research Council (NRC) of the US National Academies of Science, high-throughput in vitro tests will provide evidence for alterations in "toxicity pathways" as the best possible method of large scale toxicity prediction. The challenges to implement this proposal are enormous, and provide much room for debate. While many efforts address the technical aspects of implementing the vision, many questions around it need also to be addressed. Is the overall strategy the only one to be pursued? How can we move from current to future paradigms? Will we ever be able to reliably model for chronic and developmental neurotoxicity in vitro? This paper summarizes four presentations from a symposium held at the International Neurotoxicology Conference held in Xi'an, China, in June 2011. A. Li reviewed the current guidelines for neurotoxicity and developmental neurotoxicity testing, and discussed the major challenges existing to realize the NCR vision for toxicity testing. J. Llorens reviewed the biology of mammalian toxic avoidance in view of present knowledge on the physiology and molecular biology of the chemical senses, taste and smell. This background information supports the hypothesis that relating in vivo toxicity to chemical epitope descriptors that mimic the chemical encoding performed by the olfactory system may provide a way to the long term future of complete in silico toxicity prediction. S. Ceccatelli reviewed the implementation of rodent and human neural stem cells (NSCs) as models for in vitro toxicity testing that measures parameters such as cell proliferation, differentiation and migration. These appear to be sensitive endpoints that can identify substances with developmental neurotoxic potential. C. Suñol reviewed the use of primary neuronal cultures in testing for neurotoxicity of environmental pollutants, including the study of the effects of persistent exposures and/or in differentiating cells, which allow recording of effects that can be extrapolated to human developmental neurotoxicity.

Keywords: neurotoxicity testing; developmental neurotoxicity test guidelines; olfactory system; primary neuronal cultures; human neuron stem cells 


\section{Introduction.}

The founder of modern toxicology, Mateu Orfila (1787 - 1853), recognized that toxic effects on the nervous system include extreme diversities of both causes and outcomes (Orfila, 1826). The extreme diversity of cell types and cell interactions included in the nervous system, and the associated selectivity that most toxic actions show for a single or a limited number of these kinds of cells (Spencer, 2000) make predicting toxicity in this system a challenging task within the view proposed by the report "Toxicity testing in the $21^{\text {st }}$ century: A vision and a strategy" (NRC, 2007). This report advocates for a toxicity testing strategy that relies on high throughput in vitro tests sensitive to key effects on "toxicity pathways". In the era of molecular cell biology, robotics and informatics, this proposal seems a good one, but whether or not it will really answer the needs is an open question. Many meetings and symposia have focused on the results obtained in the development of methods for high throughput testing. However, there is also a need for debating the overall strategy, and for evaluating fundamental aspects of neurotoxicity studies that are relevant to the value of the testing schemas.

This article summarizes a symposium entitled Strategies and tools for preventing neurotoxicity: to test, to predict and how to do it that was presented at the Xi'an International Neurotoxicology Conference, Neurotoxicity and Neurodegeneration: Local Effect and Global Impact held on June 5-10, in Xi'an, China. The first speaker in this symposium, Dr. Abby Li, reviewed the current test guidelines for adult and developmental neurotoxicity testing, and extended on the needs and challenges involved in the transition from the present scenario to the one envisaged by the NRC $21^{\text {st }}$ century vision report. The second speaker, Dr. Jordi Llorens, defended the hypothesis that we may go beyond the NRC $21^{\text {st }}$ century proposal and also start preparing for a long term goal of a complete in silico neurotoxicity prediction. He reviewed the role of the taste and olfactory systems in toxic avoidance by living mammals to conclude that a great effort in in vivo toxicity studies is also necessary to collect a database of toxic effects related to chemical epitopes, on which the in silico modeling can be based. The two remaining talks reviewed the results from the author's own laboratories that are of highly relevance for future developments of in vitro testing strategies. One major challenge to these strategies is the need to evaluate the potential of chemicals for chronic neurotoxicity and neurodevelopmental toxicity. Dr. Sandra Ceccatelli reported on her studies using neuronal stem cells for in vitro neurotoxicity evaluation, as chemicals with preferential activity against these cells are likely to be neurodevelopmental toxicants. She showed how the phenomena of cell proliferation, differentiation and migration can be studied in these cells, and the impact of neurotoxic 
exposure on these parameters that are well recognized as critical points in neurodevelopment. The fourth speaker, Dr. Cristina Suñol, reviewed her results using neuronal cell cultures exposed to environmental pollutants for several days. Her results indicate that neuronal in vitro systems can be used not only to evaluate cytotoxic effects, but also to assess more subtle effects such as the establishment of persistent changes in synaptic transmission, more likely to be relevant for chronic neurotoxicity and neurodevelopmental toxicity. Together, the symposium contributed to the debate about how we will predict neurotoxicity and neurodevelopmental toxicity in the future.

\section{Regulatory Neurotoxicity Testing for Risk Assessment Purposes: Current and Future Approaches.}

Abby A. Li, Ph.D. Exponent Health Sciences, San Francisco, USA

Current test guidelines for standard toxicity testing, acute and subchronic neurotoxicity testing, and developmental neurotoxicity (DNT) testing rely primarily on behavioral and histopathology evaluations of the nervous system (Tables 1 and 2). For example, the DNT test requires an evaluation of different types of behavioral measures including gross neurologic and behavioral abnormalities, motor activity, startle habituation and neuropathologic evaluations including morphometric measures (Table 2). This test is designed to assess different types of behavioral functions and evaluate histopathology sections throughout the central and peripheral nervous system. The study designs require three treatment groups and one control group with the highest dose level inducing systemic toxicity, but not death or severe suffering of the animals.

EPA's Neurotoxicity Risk Assessment Guidelines (USEPA, 1998) and OECD's guidance document on neurotoxicity testing (OECD, 2004) provide guidance on interpreting functional and morphologic changes. Specifically, changes in behavior may be adverse but are not necessarily the result of a direct effect on the nervous system, especially when testing chemicals near the maximum tolerated dose (USEPA, 1998). In addition, evaluation of whether behavioral changes are adverse should take into consideration the consistency of the pattern of effects. For example, in evaluating results from the functional observational battery (FOB), which can include more than 30 different measures of normal and/or abnormal behaviors, "the risk assessor should be aware of the potential for a number of false positive statistical findings in these studies because of the large number of endpoints customarily included in the FOB" (USEPA, 1998). OECD (2004) and USEPA (1998) recommend that the assessment of neurotoxicity should incorporate a level of concern based on the type, severity, 
number, and either full or partial reversibility of the effect(s). A pattern or cluster of related effects generally causes a higher level of concern than individual or unrelated effects. However, the observation of some specific endpoints, even those of limited duration in time (e.g., body tremors, convulsions) may be sufficiently important depending on dose level and relative sensitivity compared to other toxicity endpoints (OECD, 2004).

Different regulatory agencies have different testing requirements for neurotoxicity testing depending on chemical class, use, volume of production, or extent of exposure to humans. The USEPA Office of Pesticide Programs (USEPA OPP) requires acute and subchronic neurotoxicity testing in adults for all food use pesticides (USEPA, 2011). USEPA OPP considers the developmental neurotoxicity test (DNT) as conditionally required based on evidence of (1) treatment-related neurological effects in adult or developing animals, (2) a causative association between exposures and adverse neurological effects in human epidemiological studies, or (3) a mechanism of action that is associated with adverse effects on the development of the nervous system (e.g., structure activity relationship to known neurotoxicants, altered neuroreceptor or neurotransmitter responses) (USEPA, 2011).

For other regulatory agencies and types of chemicals, the first animal data indicating a need for neurotoxicity assessment are provided by standard toxicity studies where functional and/or histopathological information is gathered on all major organ systems, including the nervous system (Table 2; OECD, 2004). When there are indications of possible neurotoxic effects, additional endpoints may be included in the initial standard tests(s), or the results may trigger the need for adult and/or developmental neurotoxicity tests (OECD, 2004).

A tiered approach to testing is consistent with the report titled Toxicity Testing in the $21^{\text {st }}$ Century (NRC, 2007, p.28), which concluded that the intensity and depth of testing should focus resources on the evaluation of the more sensitive adverse effects of exposures of greatest concern rather than on full characterization of all adverse effects irrespective of relevance for risk-assessment and risk-management needs. It also stated that testing strategies need to balance the depth and breadth of testing with animal welfare concerns and expenditure of money and time on testing and regulatory review. This tiered testing approach depends on a first tier of testing that adequately and rapidly screens for a large number of chemicals for all endpoints of importance including neurotoxicity in adult and developing animals.

Current test methods for neurotoxicity testing, especially DNT, are resource intensive and use a large numbers of animals (at least 900 animals for DNT studies). Draft OECD guidelines for an extended 1-generation study include DNT endpoints as a 
means of combining elements of DNT testing with a reproduction study (OECD, 2010, Table 2). In addition, some of the behavioral endpoints may have high inherent or experimental variability (Raffaele et al., 2008). This variability is a result, in part, to the fact that 20-40 pups/litter/dose level must be tested at specific ages over a span of 4-5 days depending on when the pups from all litters are born. These studies could be conducted using staggered start dates for different replicates, but extending testing across weeks or months can contribute further to variability of the data. Thus, approaches that might improve screening of potential neurotoxicants could aid in prioritizing chemicals for further neurotoxicity testing.

The new NRC $21^{\text {st }}$ century vision emphasizes the use of high-throughput assays, computational tools, and scientific advances in understanding perturbations of cellular-response networks - complex interaction of genes, proteins, and small molecules — that lead to adverse health effects (Judson et al., 2010; Martin et al., 2011; NRC, 2007). Other approaches include the use of a battery of medium-throughput in vitro cell culture assays to model key events of brain development such as proliferation, differentiation, migration, neurite growth, synaptogenesis, myelination, and apoptosis. Additionally, alternative nonmammalian species (e.g., zebrafish, C. elegans) can be used to screen for chemical effects on an intact rapidly developing nervous system (Coecke et al., 2007; Lein et al., 2005, 2007; MacPhail et al., 2009; Radio and Mundy, 2008; Ton et al. 2006).

There are significant challenges in realizing this vision for toxicity testing. These include (1) identifying and validating batteries of tests, (2) separating adaptive or negligible perturbations from those that are adverse, (3) determining if all sensitive windows of development are adequately tested, and (4) understanding the relevance of dose-response relationships in in vitro systems to human exposures (NRC, 2007). As a first step towards identifying and comparing different batteries of in vitro tests for purposes of screening chemicals for prioritization, Crofton et al. (2011) developed a set of best practices or principles to facilitate comparison of new approaches across different laboratories. These general principles still need to be translated into specific criteria. At present, comparisons across laboratories are hampered by use of different testing conditions in different laboratories; use of too few (e.g., <5) or different positive and negative controls for specific endpoints; limited comparisons between endpoints measured and reliable measures of cytotoxicity; too few doses (e.g., <10) or insufficient number of replicates (e.g., <3); and inadequate reporting of test material purity, testing conditions, and results. Protocol optimization and standardization are essential first steps that are required before methods can be validated for use in a regulatory testing scheme (Crofton et al., 2011). In addition, specific criteria based on these general 
principles outlined by Crofton et al. (2011) should be inclusion criteria for any exploratory data-mining computational efforts. At present, it is premature to make any conclusions about which battery of in vitro methods, if any, will improve developmental neurotoxicity testing.

An integral part of the NRC $21^{\text {st }}$ century vision that has not been given sufficient attention is the use of targeted testing to complement toxicity pathway tests and to support toxicity evaluation for risk assessment purposes. Targeted in vivo or in vitro testing can be used to investigate and clarify uncertainties with mechanisms, metabolites, target tissue reactions, and cellular responses at the genomic level (NRC, 2007). These could involve limited types and shorter duration in vivo studies. For instance, existing toxicity-test systems using standard animals, as well as transgenic species, could include toxicogenomic endpoints that are considered reliable early predictors of toxicity. Plunkett et al. (2010) proposed a flexible integrated approach that targets testing based on physiochemical properties, knowledge of potential mode of action of the specific or related chemicals, biological profiling using in silico methods, and information of potential exposures. Such an approach, if focused on the most sensitive endpoints based on dose-response comparisons, is consistent with the NRC $21^{\text {st }}$ century vision and provides an evolutionary path forward to gradually adopt new methods.

Integrated testing approaches that use current and new approaches for neurotoxicity testing have the potential of reducing the number of redundant animal tests, while increasing the breadth of testing associated with those animal tests. Although significant challenges lie ahead for validation of new approaches and interpretation of the results, new technologies have the potential of assessing endpoints that are more specific to the developing nervous system, including obligatory "precursor" events that are predictive of neurologic diseases that are difficult to screen using behavioral tests in animal models (e.g., ADHD, autism). In addition, medium or high throughput methods have the potential to improve the grouping of chemicals by similar mode of action instead of relying exclusively on chemical structure. This can reduce animal testing by providing scientific basis for selecting a representative chemical to be tested for a group of chemicals with similar mode of action. Finally, if properly validated, these approaches will improve screening of chemicals for potential neurotoxicity and focus additional testing resources towards those chemicals of greatest concern. 


\section{Toward Neurotoxicity Testing in the $22^{\text {nd }}$ Century: A Vision, a Taste or a Smell?}

Jordi Llorens. Department of Physiological Sciences II, Universitat de Barcelona IDIBELL, Hospitalet de Llobregat, Spain

Traditional methods for neurotoxicity prediction and prevention have been based on animal testing. Now, the substitution of animal testing for in vitro testing is on the table for debate, but there is no much debate on the need for testing. And what we really need at the end is to prevent neurotoxic damage by predicting it; testing is only a means of obtaining data useful for prediction. So why not postulate that the ultimate long term goal, the one for the $22^{\text {nd }}$ century, should be to have the capacity to fully predict toxicity in silico?. If we aim at this long-term goal, one relevant question is whether current efforts for developing high throughput testing system are also in the right direction for this objective, or we need to devote additional efforts in other directions. This part of the symposium discussed the strategy used by mammals for toxicity avoidance, with the purpose of learning about a quite successful strategy from which some useful ideas may emerge. No long ago, we prevented toxicity as wild mammals using our sensory systems to avoid ingestion of toxic foods. Many factors now make this biological system for (neuro)toxicity prevention an insufficient one. These include 1) dermal and inhalation routes of exposure are increasingly significant in contrast to the oral route that likely predominated toxic exposure during evolution; 2) high concentrations of lipophilic compounds are likely more abundant in our present environment than in common foods of the pre-Neolithic times; 3) toxic molecules may belong to new classes of compounds, differing from anything we have encountered previously during evolution; 4) increased life expectancy makes the long term, delayed or cumulative effects much more important. This is an aspect in which the nervous system emerges as the main one of concern because of its limited capacity for regeneration. These factors make it important that toxicological sciences help us to prevent toxic damage, but they do not invalidate the logic behind the biological strategy, which has been quite successful. Modern biological sciences provide an overall understanding of some major features of sensory physiology, and we can hypothesize how they act in toxicity prevention and perhaps learn some useful lessons.

Chemical components included in food are analyzed by two major sensory systems, the taste system in the oral cavity and the olfactory system by the retronasal route. In the taste system, each taste cell selectively responds to one of a few basic taste modalities (for a review, see Chandrashekar et al., 2006), five of which are currently recognized: salty, sour, bitter, sweet and umami. Some other basic tastes 
may exist, as one for lipids, but the list is quite short in any case. The bitter taste is the one that specializes in detecting toxic compounds to be avoided, although extremely salty and sour tastes also generate avoidance responses. Bitter compounds are those that bind to bitter taste receptors, a family of around thirty G-protein coupled receptors known as T2Rs (for taste-2 receptors) (Adler et al., 2000). All of these bitter receptors are expressed in the same taste cells, which act as broadly tuned bitter sensors that are wired to mediate behavioral aversion and differ from the ones mediating the other taste modalities (Mueller et al, 2005). This organization has some important implications.

First, there is one single bitter perception, we can discriminate among different concentrations of bitter compounds, but we cannot distinguish among different classes of bitter compounds. Second, our ability to detect toxic compounds by their taste depends on genetic information encoded in the bitter receptors. Third, the bitter receptors are likely adjusted to bind compounds with a relevant toxic role during our evolutionary history. Fourth, the small number of existing T2Rs, 25 in humans, implies that, even if broadly tuned, only a few hundred bitter compounds are probably detectable. Thus, the bitter taste system seems adapted to provide a fast warning signal for bulk content of toxins in food, but with no discrimination ability (Yarmolinsky et al., 2009).

In contrast to the few modalities existing for taste perception, there are no basic modalities for olfactory perception. There are hundreds of different olfactory receptors (339 in humans, 913 in mice) and each of them is expressed in a specific population of olfactory neurons that will respond to chemicals binding to this particular receptor (Buck and Axel, 1991; Uchida et al., 2000; reviewed by DeMaria and Ngai, 2010). Each olfactory receptor (and therefore each class of olfactory neuron) is activated by compounds with certain molecular features (e.g., aldehydes), but is also broadly tuned for other characteristics (e.g. carbon chain length). The receptor does not recognize the entire molecule, but a part of it, a "chemical epitope", and will be activated by different molecules that have this epitope in common. Overall, the olfactory system uses several hundreds of receptors for chemical recognition to respond to an endless list of molecules, each encoded as a particular combination of chemical epitopes; it is a system for detailed chemical analysis with a very high capacity for discrimination. This capacity for discrimination can be used for toxic avoidance following rules that are learned. It is well known that mammals develop robust avoidance responses to flavors once paired with sickness (Garcia et al., 1955). Current knowledge on the physiology of the taste and smell systems indicates that the olfactory system must be the main one involved in the chemical recognition necessary for this response. 
The mammalian system for avoiding toxicity is thus based in two complementary strategies. First, the taste system is used to avoid ingestion of toxic doses of potentially harmful compounds as genetically encoded in the bitter receptors. Second, foods and drinks accepted by the taste system are submitted to a detailed chemical analysis and refused in the future if signals of disease (pain, nausea, dizziness) follow the previous ingestions.

The proposed strategy for toxicity testing based on high throughput in vitro tests sensitive to key effects on toxicity pathways will, if successful, provide a system that will share many features with the taste system. Test validation will take place of evolutionary selection, and the process will likely provide a limited number of test systems for high throughput toxicity testing, as we have a limited number of bitter taste receptors for quick rejection of toxic compounds. These systems will have limitations imposed by the currently available knowledge in the same way that bitter receptors likely recognize only toxic compounds that we have encountered in significant amounts during evolution. So we will need a second system that, like the avoidance learning system based on olfactory information, is open to learning about new toxic effects caused by new compounds that have passed the first screen. The advantage now is that any new knowledge will persist and not disappear, in the way that flavor aversions are lost when the subject dies.

Our system open to learning will necessarily cover some gaps in the biological system, including associations for chronic and delayed toxicity, but other parts can be mimicked. In particular, the "chemical epitope" approach of describing the chemical world used by the olfactory system is likely to be useful in terms of toxicity prediction, and is likely to be particularly well suited for in silico modeling. Because a few hundreds of olfactory receptors exist, perhaps a similar or a few times greater number of epitope descriptor models (from 300 to 3,000 in total) could be enough (that is, any chemical potentially interacting with our organism could be satisfactorily described as a combination of several of these possible epitopes). Recent improvements in chemical description for quantitative structure-activity relationship (QSAR) methods, as groupbased QSAR (Ajmani et al., 2009) appear to be moving in the direction of a closer resemblance to the chemical epitope recognition performed by the olfactory system.

Besides chemical description, the system will need toxicity data for pairing chemical epitopes with the toxicity outcomes. In my opinion, because they include the largest possible variety of initial targets and integrate all the necessary parameters involved in toxicity outcome, in vivo studies, using multiple species and paradigms, are likely to be the most efficient way to generate the needed knowledge, whatever the toxicity pathways linking the initial targets to the final toxic effect. Although we know 
about the many kinetic and dynamic variables involved in any toxic effect, it is compelling to recognize that the natural avoidance system simply pairs olfactory chemical analysis with a few apical indices of illness. Also, the nervous system may include many unique toxicity pathways, but its singularity for toxicity more likely relies on the diversity of initial targets it contains.

The above information leads me to express two proposals. The first major proposal is that in addition to developing tests for high throughput toxicity testing we need to make a huge effort of data collection, including much in vivo data, to approach the dreamed end goal of in silico toxicity prediction. In vivo testing represents the evaluation of an unbeatable diversity of potential targets that we need to take into account in our efforts to toxicity prediction. It is obvious that we cannot test all chemicals in live mammals, but there is a lot that we need to learn from in vivo models, including mammalian species. A relevant effort generating useful data is that of the pharmaceutical industry in both pre-clinical tests and clinical trials; how we get to use this information is a relevant question. Although pharmaceuticals are intentionally designed to minimize their toxicity and are thus a biased sample of the chemical universe, the discovery of unexpected toxic effects associated with new chemical epitopes may provide useful cues for subsequent in silico toxicity prediction.

Importantly, if toxic effects are found at the clinical trial level, after extensive pre-clinical testing, this indicates human-specific effects.

A second proposal is that we may want to use the chemical epitope logics for data collection and analysis. In most cases, toxicity evaluation is driven by chemical use and its known toxicity. That is, toxicity studies focus on compounds that we are significantly exposed to or that are identified as potentially toxic at dose levels similar to exposure levels. This seems a sound way of using the available resources. However, I propose that we also need to devote a significant amount of resources to evaluate toxicity for families of chemicals defined by a particular chemical epitope, as this may generate knowledge particularly useful for the final goal of in silico prediction. One example of the potential interest of this approach is the work by LoPachin et al. (2008) raising the hypothesis that conjugated $\alpha, \beta$-unsaturated carbonyl derivatives (that is, chemicals containing this particular chemical epitope) are particularly prone to cause neurotoxicity by presynaptic damage via protein adduct formation. Another example is the study on the neurotoxic effects of nitriles (compounds with a cyano, R-CN, group) on the sensory and central nervous systems. Many nitriles are known to cause acute toxicity through cyanide release by xenobiotic metabolism enzymes, a toxic endpoint already addressed by some modeling studies (Grogan et al., 1992). The work by several groups, including ours, has demonstrated that several nitriles cause a variety of 
neurotoxic effects, such as degeneration of specific populations of neurons in the central nervous system (Boadas-Vaello et al., 2005; Seoane et al., 2005) and degeneration of sensory systems including the olfactory (Genter et al., 1992), auditory (Gagnaire et al., 2001) and vestibular systems (Llorens et al., 1993; Balbuena and Llorens, 2001, 2003). We have observed that vestibular toxicity is not related to cyanide release (Boadas-Vaello et al., 2007, 2009) and is associated with strict structural requirements (Balbuena and Llorens, 2003) that may depend on the animal species, as the case of trans-crotononitrile, which causes neuronal degeneration in the rat (Boadas-Vaello et al., 2005; Seoane et al., 2005) and vestibular toxicity in the mouse (Saldaña-Ruíz et al., 2012). An evaluation of 19 similar nitriles for vestibular toxicity in the mouse has resulted in 5 positive nitriles (Saldaña-Ruíz et al., 2012). We hypothesize that the nitrile group has a diverse but limited number of neurotoxic effects, and that the structural motifs leading to each one of them will be amenable to modeling. That is, we will probably be able to define a small number of chemical epitopes containing the nitrile group and causing different neurotoxic effects, and to model the relationship between chemical structure and neurotoxicity for all of them. As these relationships most often depend on chemical-to-protein interaction, speciesspecific differences emerge, but these will be included in the models.

Similarly to the above examples, any given group or chemical epitope may have a diverse but probably limited number of toxic effects. Detection and characterization of all these effects for future modeling and prediction looks like an enormous task. To begin with, one would like to have an estimate of its size, as imprecise as it may be. My hypothesis is that the numbers provided by the olfactory system, from several hundreds to a few housand epitopes, provide such a rough estimate and it does not look impossible in a century perspective.

\section{Neurotoxicity Testing: The Challenge Of In Vitro Alternatives}

Sandra Ceccatelli. Department of Neuroscience, Karolinska Institutet, Stockholm, Sweden

The complex anatomical and physiological organization of the nervous system, with different cell types forming networks essential to maintain all integrated functions, cannot be reproduced in in vitro systems. However, the use of multiple in vitro models, including primary cultures of neurons obtained from specific brain areas, cell lines of different origin, co-culture systems with neuronal and glial cells, have been shown to be valuable in neurotoxicological research. The nervous system is particularly sensitive to 
alterations of the microenvironment occurring during development, and there is growing evidence pointing to developmental exposure to chemicals as possible cause of nervous system disorders (Landrigan et al., 2005). Well characterized in vitro model systems are therefore needed for large scale "animal-free" screening of chemicals and pharmaceutical drugs for the assessment of neurotoxic effects, with special focus on developmental neurotoxicity (DNT).

In recent years, we have successfully implemented the use of neural stem cells (NSCs) as models for in vitro DNT tests (Sleeper et al., 2002; Tamm et al., 2004, 2006, $2008 a, b, c)$. NSCs play a critical role in the developing embryonic nervous system through to adulthood, where the ability for self-renewal appears to be important for normal functions, such as learning, memory, and response to injuries. Using NSCs from rodents and humans, we have investigated the effects of environmental contaminants, such as methylmercury $(\mathrm{MeHg})$ and non-dioxin-like polychlorinated biphenyls (PCBs) on survival and differentiation potential.

We prepare primary cultures of NSCs from embryonic cortices from timedpregnant Sprague-Dawley rats (Harlan Laboratories, The Netherlands) at E15 (the day of copulatory plug was defined as E0). Primary embryonic/fetal human neural cells, derived from fetuses terminated at gestational week (GW) $6-16$, are cultured as neurospheres or dissociated cells. To study proliferation and survival, cells are maintained in a medium enriched with appropriate growth factors to keep cells in an undifferentiated and proliferative state. Studies on spontaneous differentiation and migration capabilities are performed in the absence of growth factors

Briefly, our results show that NSCs are highly sensitive to neurotoxicants, as compared to other neural cell types (Tofighi et al. 2011a). MeHg induces apoptosis via Bax-activation, cytochrome $\mathrm{c}$ translocation and caspases, as well as calpain activation. Conversely, the selected PCBs tested (PCB 153 and 180) induce cell death via a lysosome-dependent pathway. Remarkably, developmental exposure to $\mathrm{MeHg}$, at concentrations comparable to those found in the cord blood of the general population in many countries, inhibits spontaneous neuronal differentiation of NSCs via Notch signaling (Tamm et al. 2006; 2008a). On the contrary, nanomolar concentrations of PCB 180 and 153 increased spontaneous neuronal differentiation, as shown by the increase in Tuj 1 positive cells, with a concomitant decrease in proliferating cells, as detected by EdU-staining. Combined exposures to PCBs and $\mathrm{MeHg}$ resulted in a synergistic interaction with regard to the induction of apoptotic cell death and an antagonistic interaction on spontaneous neuronal differentiation of NSCs (Tofighi et al. 2011b). 
With regard to human NSCs, after several tests, we came to the conclusion that for proliferation or differentiation assays, dissociated cells provide the most reliable results. For migration assays instead, we implemented intact neurospheres. Cells from different developmental stages cultured as neurospheres were exposed to a range of $\mathrm{MeHg}$ concentrations (2.5nM, 10nM, 25nM, 100nM) and alterations in differentiation and migration were examined and compared to untreated control-cultures processed in parallel. Exposure to $\mathrm{MeHg}(10-25 \mathrm{nM})$ resulted in a significant decrease in neuronal differentiation (Tuj1 quantification), similar to what was observed in rodent NSCs (Edoff et al. unpublished).

In conclusion, it appears that NSCs are promising in vitro models for neurotoxicity studies and that parameters such as cell proliferation, differentiation and migration are sensitive endpoints to identify substances with developmental neurotoxic potential.

\section{Cell-Based Methods for Predicting Neurotoxicity of Environmental Pollutants}

Cristina Suñol. Department of Neurochemistry and Neuropharmacology. Institut d'Investigacions Biomèdiques de Barcelona. IIBB-CSIC-IDIBAPS; CIBERESP. Barcelona. Spain. E-mail: csenqi@iibb.csic.es

More than 200 chemicals have been reported to produce neurotoxicity in humans and some of them. are permanent organic pollutants and bioaccumulate in food chains, contaminating the food and, therefore, posing a risk for human health (Grandjean and Landrigan, 2006). This is the case, for example, with organochlorine pesticides, PCBs and methylmercury. Neurotoxicity is usually manifested as alterations of behavior including cognitive, motor and learning-memory processes, which are often a consequence of alterations in GABAergic and glutamatergic neurotransmission, and of neurodegenerative processes. Recent studies demonstrated the presence of environmental contaminants in human samples, including those from children. In fact, persistent organochlorine pesticides and methylmercury have been found with high frequency in placenta, cord blood and children's adipose tissue (López-Espinosa et al., 2007, 2008; Ramon et al., 2011; Younglai et al., 2002). Furthermore, epidemiological studies found correlations between exposure to these neurotoxicants and neurological deficits in children (Grandjean and Landrigan, 2006; Morales et al., 2008; Freire et al., 2010). Therefore, toxicity testing is needed for predicting neurotoxicity of low- and longterm exposure to environmental pollutants. To this aim, we use primary neuronal cultures as experimental models for neurotoxicity testing. 
Primary cultures of mouse cerebellar granule cells consist mainly of glutamatergic neurons (> $90 \%$ ) with a small proportion of GABAergic neurons ( $6 \%$ ), whereas primary cultures of mouse cortical neurons contain both GABAergic, cholinergic and glutamatergic neurons as the main neuronal types (Solà et al., 2011; Sonnewald et al., 2004; Suñol et al., 2008). Moreover, the functional expression of GABA-A, glutamate, glycine and cholinergic receptors, as well as neuronal transporters for glutamate and GABA and enzymes for the synthesis/degradation of these neurotransmitters is well documented in the literature. It has been reported that neurons develop in vitro faster than in vivo (Yu et al., 1984) acquiring characteristics of GABA and glutamate neurotransmisión within 1 - 2 weeks in culture (Yu et al., 1984; Frandsen and Schousboe, 1990; Griffiths et al., 1997; Hogberg et al., 2010). Therefore, cells can be exposed to chemicals either at maturation (after 1 week in vitro) or during their differentiation to study neurodevelopmental toxic effects/mechanisms of toxicity. We present here our recent findings on the prolonged effects of environmental persistent pollutants on GABA and glutamate neurotransmission, on redox homeostasis and on cytoskeletal proteins in primary rodent cultured neurons.

Dieldrin is one of the compounds included in the Stockholm Convention on Persistent Organic Pollutants because of its toxicity and high persistence in the environment. Exposure of primary cultures of cortical neurons to dieldrin for 6 days in vitro (div) resulted in cytotoxic effects at concentrations $>200 \mathrm{nM}$. Lower non-cytotoxic concentrations of dieldrin (e.g., $60 \mathrm{nM}$ ) significantly decreased $\mathrm{GABA}_{\mathrm{A}}$ receptor function as measured by the reduced binding of $\left[{ }^{35} \mathrm{~S}\right] \mathrm{TBPS}$ and reduced GABA-induced $\mathrm{Cl}^{-}$influx. This reduction of GABA neurotransmission is in agreement with the primary mechanisms of dieldrin neurotoxicity, namely the inhibition of the $\mathrm{GABA}_{A}$ receptor. Accordingly, this in vitro inhibition will be predictive of an excitatory syndrome that eventually can lead to the induction of convulsions in mammals. However, this continuous inhibiton of the $\mathrm{GABA}_{\mathrm{A}}$ receptor might also induce a synaptic scaling compensatory effect, scaling down excitatory glutamate receptors to protect the CNS system from imbalance of excitatory-inhibitory neurotransmission (Watt et al., 2000). We found that prolonged in vitro exposure of cultured neurons to dieldrin resulted in decreased functionality of ionotropic NMDA and type I metabotropic mGluR5 glutamate receptors. This decreased activity was accounted for by internalization and downregulation of NMDA and mGluR5 receptors, respectively (Babot et al., 2005; Briz et al., 2010). Figure 1 summarizes the effects of prolonged exposure to a non-toxic concentration of dieldrin on glutamatergic neurotransmission in primary cultures of cortical neurons. From these findings we conclude that prolonged exposure to dieldrin in vitro led to reduced performance of the $\mathrm{GABA}_{A}$ and glutamate receptors, which may 
be predictive of deleterious consequences in vivo because NMDA receptor is involved in memory and learning processes. In fact, behavioral and learning deficits have been observed in monkeys chronically dosed with dieldrin (Smith et al., 1976). On the other hand, dieldrin has been reported to be an endocrine disruptor. In neurons, dieldrin inhibited $\left[{ }^{3} \mathrm{H}\right]$ estradiol binding to the neuronal estrogen receptor $(E R)$ and induced the activation of the membrane ER downstream signaling pathways Akt and ERK. In addition, exposure to dieldrin for 6 div down-regulates the expression of neuronal ERalpha (Briz et al., 2011). These effects of dieldrin on ER levels and activation might contribute to cognitive and behavioral deficits (Schantz and Widholm, 2001).

Methylmercury is a neurotoxic agent to which the population is exposed through consumption of predator fishes that have bioaccumulated it (McDowell et al., 2004; Ramon et al., 2011). Targets of methylmercury in the CNS are the granule cell layer of the cerebellum and the cortex visual area. In cultured cerebellar granule cells, acute exposure to methylmercury inhibits neuronal glutamate uptake, increases extracellular glutamate, produces reactive oxygen species and increases intracellular calcium (Allen et al., 2001; Fonfría et al., 2005; Gassó et al., 2001). Methylmercury-induced cell death was concentration- and time-dependent in cerebellar granule cells. Prolonged exposure of cerebellar granule cells during maturation in vitro to methylmercury resulted in reduced glutathione peroxydase (GPx) activity before methylmercury induced neuronal death, which was related to enhanced susceptibility to hydrogenperoxide. Accordingly, lipid peroxidation was produced, which was reversed by the antioxidant probucol. At the same time, probucol increased GPx activity. Likewise, overexpression of GPx-1 in cultured cerebellar granule cells resulted in neuroprotection against methylmercury-induced cell death (Farina et al., 2009). Figure 2 summarizes some of these findings. On the other hand, no effects were found in reduced and oxidized glutathione levels, glutathione reductase and catalase activity, nor on neuronal glutamate uptake or intracellular calcium, after using the same protocol for methylmercury exposure (Farina et al., 2009). Altogether, these results suggest that oxidative stress driven by inhibition of glutathione peroxidase may be one of the first hallmarks of methylmercury-induced neurotoxicity in the development of cerebellar granule cells, a finding also observed in mice exposed to methylmercury (Franco et al., 2009). Under similar in vitro exposure conditions, an increase in non-phosphorylated cofilin and translocation of non-phosphorylated cofilin from the cytosol to the mitochondria was also found by means of a proteomic approach (Vendrell et al., 2010). The balance of cofilin phosphorylation / non-phosphorylation forms regulates actin dynamics and facilitates actin filament turnover, which are responsible for neuron 
shape change, migration, polarity formation and regulation of synaptic structures and function. Therefore, an alteration of the complex regulation of the cofilin phosphorylation/dephosphorylation pathway could be envisaged as an underlying mechanism compatible with reported signs of methylmercury-induced neurotoxicity such as those observed in vivo (apoptosis, cell migration deficits).

In conclusion, cell-based methods using primary neuronal cultures of different cell types (cortical neurons and cerebellar granule neurons) constitute good models for testing the neurotoxicity of environmental pollutants. Non-cytotoxic concentrations of the agents have been used and this allowed us to determine their effects on neural functions in the absence of general toxicity. Following the recommendations of the US National Academies, I have shown that these models may be incorporated into a predictive strategy based on in vitro toxicity assays, because they are able to predict at a cellular level effects that can be extrapolated to effects on individuals.

\section{Conclusion.}

Toxicity prediction is particularly challenging in the case of chronic neurotoxicity and neurodevelopmental toxicity effects, and these areas place a particular challenge to the NRC $21^{\text {st }}$ century vision strategy. In the symposium reported here, a comparison was presented on the current and proposed approaches to neurotoxicity testing, highlighting critical questions that need to be addressed and are of particular relevance in the transition from the present to the future approaches. While recognizing the difficulties ahead, this talk provided a hopeful view of significantly ameliorating our testing strategies for neurotoxicity prediction. An alternative view was presented the symposium, defending that the goal of a complete in silico neurotoxicity prediction is possible in the very long term, but that approaching this goal will require the use of in vivo studies to collect large amounts of toxicity data for chemical epitopes. Within the predominant strategy of moving towards an increasing role for in vitro test systems, we need to know how subtle effects on brain function and development resulting from low dose or chronic exposure can be evaluated in these systems. The results discussed on this synopsis on neural stem cells and on long-term neuronal culture effects showed that in vitro systems can go beyond cytotoxicity assessment, and be useful in evaluating adverse functional effects likely relevant to chronic and developmental neurotoxicity. Evolving these contributions into practical tools for toxicity prediction is a challenging endeavor. 


\section{ACKNOWLEDGEMENTS}

This research was supported by grants from the Spanish Ministries of Health and of Science and Innovation [PI 061212, PI 10/0453 and BFU2009-06945], the Generalitat de Catalunya [2009/SGR/214], and the Swedish Research Councils VR and FORMAS.

\section{REFERENCES}

Adler E, Hoon MA, Mueller KL, Chandrashekar J, Ryba NJ, Zuker CS. A novel family of mammalian taste receptors. Cell 2000; 100:693-702.

Ajmani S, Jadhav K, Kulkarni SA. Group-based QSAR (G-QSAR): Mitigating interpretation challenges in QSAR. QSAR Comb Sci 2009: 28: 36-51.

Allen JW, Mutkus LA, Aschner M. Methylmercury-mediated inhibition of 3H-D-aspartate transport in cultured astrocytes is reversed by the antioxidant catalase. Brain Res. 2001; 902: 92-100.

Babot Z, Vilaró MT, Suñol C. Long-term exposure to dieldrin reduces $\mathrm{GABA}_{\mathrm{A}}$ and NMDA receptor function in primary cultures of mouse cerebellar granule cells. $J$ Neurosci Res 2007; 85: 3687 -95

Balbuena E, Llorens J. Behavioural disturbances and sensory pathology following allylnitrile exposure in rats. Brain Res 2001; 904: 298-306.

Balbuena E, Llorens J. Comparison of cis- and trans-crotononitrile effects in the rat reveals specificity in the neurotoxic properties of nitrile isomers. Toxicol Appl Pharmacol 2003; 187: 89-100.

Boadas-Vaello P, Riera J, Llorens J. Behavioral and pathological effects in the rat define two groups of neurotoxic nitriles. Toxicol Sci 2005; 88: 456-66.

Boadas-Vaello P, Jover E, Díez-Padrisa N, Bayona JM, Llorens J. Differential role of CYP2E1-mediated metabolism in the lethal and vestibulotoxic effects of ciscrotononitrile in the mouse. Toxicol Appl Pharmacol 2007; 225: 310-17.

Boadas-Vaello P, Jover E, Saldaña-Ruíz S, Soler-Martín C, Chabbert C, Bayona JM, Llorens J. Allylnitrile metabolism by CYP2E1 and other CYPs leads to distinct lethal and vestibulotoxic effects in the mouse. Toxicol Sci 2009; 107: 461-72

Briz V, Galofré M, Suñol C. Reduction of glutamatergic neurotransmission by prolonged exposure to dieldrin involves NMDA receptor internalization and metabotropic glutamate receptor 5 down-regulation. Toxicol Sci. 2010; 113: 13849.

Briz V, Molina-Molina JM, Sánchez-Redondo S, Fernández MF, Grimalt JO, Olea N, Rodríguez-Farré E, Suñol C. Differential estrogenic effects of the persistent organochlorine pesticides dieldrin, endosulfan and lindane in primary neuronal cultures. Toxicol Sci 2011; 120: $413-27$ 
Buck L, Axel R. A novel multigene family may encode odorant receptors: a molecular basis for odor recognition. Cell 1991; 65: 175-87.

Chandrashekar J, Hoon MA, Ryba NJ, Zuker CS. The receptors and cells for mammalian taste. Nature. 2006; 444:288-94.

Coecke S, Goldberg AM, Allen S, Buzanska L, Calamandrei G, Crofton K, et al. Workgroup report: Incorporating in vitro alternative methods for developmental neurotoxicity into international hazard and risk assessment strategies. Environ Health Perspect 2007; 115: 924-31.

Crofton KM, Mundy WR, Lein PJ, Bal-Price A, Coecke S, Seiler AE, Knaut H, Buzanska L, Goldberg A. Developmental neurotoxicity testing: recommendations for developing alternative methods for the screening and prioritization of chemicals. ALTEX 2011; 28:9-15.

DeMaria S, Ngai J. The cell biology of smell. J Cell Biol 2010; 191: 443-52.

Farina M, Campos F, Vendrell I, Berenguer J, Barzi M, Pons S, Suñol C. Probucol increases glutathione peroxidase-1 activity and displays long-lasting protection against methylmercury toxicity in cerebellar granule cells. Toxicol Sci. 2009; 112: $416-26$.

Fonfría E, Vilaró MT, Babot Z, Rodríguez-Farré E, Suñol C. Mercury compounds disrupt neuronal glutamate transport in cultured mouse cerebellar granule cells. $J$ Neurosci Res 2005; 79: 545 - 53.Frandsen A, Schousboe A. Development of excitatory amino acid induced cytotoxicity in cultured neurons. Int J Dev Neurosci. 1990; 8: 209-16.

Freire C, Ramos R, Lopez-Espinosa MJ, Díez S, Vioque J, Ballester F, Fernández MF. Hair mercury levels, fish consumption, and cognitive development in preschool children from Granada, Spain. Environ Res. 2010; 110: 96-104.

Gagnaire F, Marignac B, Ban M, Langlais C. The ototoxic effects induced in rats by treatment for 12 weeks with 2-butenenitrile, 3-butenenitrile and cis-2pentenenitrile. Pharmacol Toxicol 2001; 88: 126-34.

Garcia J, Kimeldorf DJ, Koelling RA. Conditioned aversion to saccharin resulting from exposure to gamma radiation. Science 1955; 122: 157-8.

Gassó S, Cristòfol RM, Selema G, Rosa R, Rodríguez-Farré E, Sanfeliu C. Antioxidant compounds and $\mathrm{Ca} 21$ pathway blockers differentially protect against methylmercury and mercuric chloride neurotoxicity. J Neurosci Res 2001; 66: 135-45. 
Genter MB, Llorens J, O'Callaghan JP, Peele DB, Morgan KT, Crofton KM. Olfactory toxicity of $B, B^{\prime}$-iminodipropionitrile (IDPN) in the rat. J Pharmacol Exp Ther 1992; 263: 1432-39.

Grandjean P., Landrigan PJ. Developmental neurotoxicity of industrial Chemicals. Lancet 2006; 368: 2167-78. Griffiths R, Malcolm C, Ritchie L, Frandsen A, Schousboe A, Scott M, Rumsby P, Meredith C. Association of c-fos mRNA expression and excitotoxicity in primary cultures of mouse neocortical and cerebellar neurons. J Neurosci Res. 1997; 48: 533-42.

Grogan J, DeVito SC, Pearlman RS, Korzekwa KR. Modeling cyanide release from nitriles : prediction of cytochrome P450 mediated acute nitrile toxicity. Chem Res Toxicol 1992; 5: 548-52.

Hogberg HT, Kinsner-Ovaskainen A, Coecke S, Hartung T, Bal-Price AK. mRNA expression is a relevant tool to identify developmental neurotoxicants using an in vitro approach. Toxicol Sci. 2010;113: 95-115.

Judson RS, Houck KA, Kavlock RJ, Knudsen TB, Martin MT, Mortensen HM, Reif DM, Rotroff DM, Shah I, Richard AM, Dix DJ. In vitro screening of environmental chemicals for targeted testing prioritization: the ToxCast project. Environ Health Perspect 2010;118: 485-92

Landrigan PJ, Sonawane B, Butler RN, Trasande L, Callan R, Droller D. Early environmental origins of neurodegenerative disease in later life. Environ Health Perspect 2005; 113:1230-3.

Lein P, Silbergeld E, Locke P, Goldberg AM. In vitro and other alternative approaches to developmental neurotoxicity testing (DNT). Environ Toxicol Pharmacol 2005; 19:735-44.

Lein P, Locke P, Goldberg A. Meeting report: Alternatives for developmental neurotoxicity testing-Test-Smart developmental neurotoxicology. Environ Health Perspect 2007; 115:764-8.

Llorens J, Demêmes D, Sans A. The behavioral syndrome caused by 3,3'iminodipropionitrile and related nitriles in the rat is associated with degeneration of the vestibular sensory hair cells. Toxicol Appl Pharmacol 1993; 123: 199-210.

LoPachin RM, Barber DS, Gavin T. Molecular mechanisms of the conjugated alpha,beta-unsaturated carbonyl derivatives: relevance to neurotoxicity and neurodegenerative diseases. Toxicol Sci 2008; 104: 235-49.

Lopez-Espinosa MJ, Granada A, Carreño J, Salvatierra M, Olea-Serrano F, Olea N. Organochlorine pesticides in placentas from southern Spain and some related factors. Placenta 2007; 28: 631-8. 
Lopez-Espinosa MJ, Lopez-Navarrete E, Rivas A, Fernandez MF, Nogueras M, Campoy C, Olea-Serrano F, Lardellib P, Olea N. Organochlorine pesticide exposure in children living in southern Spain. Environ Res 2008; 106: 1-6

MacPhail RC, Brooks J, Hunter DL, Padnos B, Irons TD, Padilla S. Locomotion in larval zebrafish: Influence of time of day, lighting and ethanol. Neurotoxicology 2009; 30: 52-8.

Martin MT, Knudsen TB, Reif DM, Houck KA, Judson RS, Kavlock RJ, Dix DJ. Predictive Model of rat reproductive toxicity from toxcast high throughput screening. Biol Reprod. 2011; 85: 327-39.

McDowell MA, Dillon CF, Osterloh J, Bolger PM, Pellizzari E, Fernando R, Montes de Oca R, Schober SE, Sinks T, Jones RL, Mahaffe KR. Hair mercury levels in U.S. children and women of childbearing age: reference range data from NHANES 1999-2000. Environ Health Perspect 2004; 112: 1165.

Morales E, Sunyer J, Castro-Giner F, Estivill X, Julvez J, Ribas-Fitó N, Torrent M, Grimalt JO, de Cid R. Influence of glutathione S-transferase polymorphisms on cognitive functioning effects induced by $p, p^{\prime}-D D T$ among preschoolers. Environ Health Perspect. 2008; 116: 1581-5.

Mueller KL, Hoon MA, Erlenbach I, Chandrashekar J, Zuker CS, Ryba NJ. The receptors and coding logic for bitter taste. Nature 2005; 434:225-9.

NRC (National Research Council). Toxicity testing in the 21 st century: a vision and a strategy, The National Academies Press, Washington, D.C., 2007.

OECD. 2004. OECD series on testing and assessment Number 20: Guidance document for neurotoxicity testing. Available at: http://www.oecd.org/officialdocuments/display documentpdf/?cote=env/jm/mono(2004)25\&doclanguage=en. Accessed August 3, 2011. Organisation for Economic Co-operation and Development.

OECD. 2010. Draft test guideline for the extended one generation reproductive toxicity. Available at: http://www.oecd.org/dataoecd/23/10/46466062.pdf. Accessed August 4, 2011. Organisation for Economic Co-operation and Development.

Orfila M. Traité des poisons tirés des regnes minéral, végétal et animal. Crochard et Gabon Eds., 3ㄹ ed. 1826, Paris, 720 pp.

Plunkett LM, Kaplan AM, Becker RA. An enhanced tiered toxicity testing framework with triggers for assessing hazards and risks of commodity chemicals. Regul Toxicol Pharmacol 2010; 58: 382-94. 
Radio, NM, Mundy, WR. Developmental neurotoxicity testing in vitro: Models for assessing chemical effects on neurite outgrowth. Neurotoxicology 2008; 29:36176.

Raffaele KC, Fisher JE Jr, Hancock S, Hazelden K, Sobrian SK. Determining normal variability in a developmental neurotoxicity test: a report from the ILSI Research Foundation/Risk Science Institute expert working group on neurodevelopmental endpoints. Neurotoxicol Teratol 2008; 30: 288-325

Ramon R, Murcia M, Aguinagalde X, Amurrio A, Llop S, Ibarluzea J, Lertxundi A, Alvarez-Pedrerol M, Casas M, Vioque J, Sunyer J, Tardon A, Martinez-Arguelles B, Ballester F. Prenatal mercury exposure in a multicenter cohort study in Spain. Environ Int 2011; 37: 597-604.

Schantz SL, Widholm JJ. Cognitive effects of endocrine-disrupting chemicals in animals. Environ Health Perspect. 2001;109: 1197-206.

Seoane A, Apps R, Balbuena E, Herrero L, Llorens J. Differential effects of transcrotononitrile and 3-acetylpyridine on inferior olive integrity and behavioural performance in the rat. Eur J Neurosci 2005; 22: 880-94.

Sleeper E, Tamm C, Frisén J, Zhivotovsky B, Orrenius S, Ceccatelli S. Cell death in adult neural stem cells. Cell Death Differ 2002; 9: 1377-8.

Solà $C$, Cristòfol R, Suñol C, Sanfeliu C. Primary cultures for neurotoxicity testing. In: .Aschner M, Suñol C, Bal-Price A, editors. Cell Culture Techniques, Neuromethods, vol. 56, 2011, 87 - 103.

Smith RM, Cunningham WL Jr, van Gelder GA, Karas GG. Deldrin toxicity and successive discrimation reversal in squirrel monkeys. (Saimiri sciureus). J Toxicol Environ Health. 1976; 1: 737-47.

Sonnewald U, Olstad E, Qu H, Babot Z, Cristòfol R, Suñol C, Schousboe A, Waagepetersen $\mathrm{H}$. First direct demonstration of extensive GABA synthesis in mouse cerebellar neuronal cultures. J. Neurochem 2004; 91: 796- 803

Spencer PS., Biological principles of chemical neurotoxicity. In: Spencer PS, Schaumburg HH, editors. Experimental and Clinical Neurotoxicology, 2 ed. New York: Oxford University Press; 2000, 3-54.

Suñol C, Babot Z, Fonfría E, Galofré M, García D, Herrera N, Iraola S, Vendrell I. Studies with neuronal cells: from basic studies of mechanisms of neurotoxicity to the prediction of chemical toxicity. Toxicol In Vitro 2008; 22: 1350 - 1355.

Tamm C, Duckworth JK, Hermanson O, Ceccatelli S. Methylmercury inhibits differentiation of rat neural stem cells via Notch signalling. Neuroreport 2008a; 19: 339-43. 
Tamm C, Sabri F, Ceccatelli S. Mitochondrial-mediated apoptosis in neural stem cells exposed to manganese. Toxicol Sci 2008b; 101: 310-20.

Tamm C, Zhivotovsky B, Ceccatelli S. Caspase-2 activation in neural stem cells undergoing oxidative stress-induced apoptosis. Apoptosis 2008c; 13: 354-63.

Tamm C, Duckworth J, Hermanson O, Ceccatelli S. High susceptibility of neural stem cells to methylmercury toxicity: effects on cell survival and neuronal differentiation. J Neurochem 2006; 97: 69-78

Tamm C, Robertson JD, Sleeper E, Enoksson M, Emgård M, Orrenius S, Ceccatelli S. Differential regulation of the mitochondrial and death receptor pathways in neural stem cells. Eur J Neurosci 2004; 19: 2613-21.

Ton C, Lin Y, Willett C. Zebrafish as a model for developmental neurotoxicity testing. Birth Defects. Res A Clin Mol Teratol 2006; 76: 553-67.

Tofighi R, Johansson C, Goldoni M, Ibrahim WN, Gogvadze V, Mutti A, Ceccatelli S. Hippocampal neurons exposed to the environmental contaminants methylmercury and polychlorinated biphenyls undergo cell death via parallel activation of calpains and lysosomal proteases. Neurotox Res 2011a; 19: 183-94.

Tofighi R, Wan Ibrahim W, Rebellato P, Andersson PL, Uhlen P, Ceccatelli S. Nondioxin like polychlorinated biphenyls interfere with neuronal differentiation of embryonic neural stem cells. Toxicol Sci 2011 b, in press.

Uchida N, Takahashi YK, Tanifuji M, Mori K. Odor maps in the mammalian olfactory bulb: domain organization and odorant structural features. Nat Neurosci 2000; 3: 1035-43.

USEPA. 1998. Guidelines for neurotoxicity risk assessment. Available at: http://www.epa.gov/raf/publications/pdfs/NEUROTOX.PDF. Accessed August 16, 2011. U.S. Environmental Protection Agency.

USEPA. 2010. OCSPP harmonized test guidelines Series 870 - health effects test guidelines.] Available at: http://www.epa.gov/ocspp/pubs/frs/publications/Test Guidelines/series870.htm. Accessed August 2, 2011. U.S. Environmental Protection Agency, Office of Chemical Safety and Pollution Prevention.

USEPA. 2011. Title 40: Protection of environment part, Part 158 Data requirements for pesticides. Available at: http://ecfr.gpoaccess.gov/cgi/t/text/textidx?c=ecfr\&sid $=$ bafa8cb23acba050f6a34a32d4003f60\&rgn=div5\&view=text\&node=40:24.0.1.1.9 \&idno $=40 \# 40: 24 \cdot 0 \cdot 1 \cdot 1 \cdot 9.6$. Accessed August 3, 2011. U.S. Environmental Protection Agency. 
Vendrell I, Carrascal M, Campos F, Abián J, Suñol C. Methylmercury disrupts the balance between phosphorylated and non-phosphorylated cofilin in primary cultures of mice cerebellar granule cells. A proteomic study. Toxicol Appl Pharmacol 2010; 242: 109 - 18.

Watt AJ, van Rossum MC, MacLeod KM, Nelson SB, Turrigiano GG. Activity coregulates quantal AMPA and NMDA currents at neocortical synapses. Neuron 2000; 26: 659-70.

Yarmolinsky DA, Zuker CS, Ryba NJ. Common sense about taste: from mammals to insects. Cell 2009; 139:234-44.

Younglai EV, Foster WG, Hughes EG, Trim K, Jarrell JF. Levels of environmental contaminants in human follicular fluid, serum and seminal plasma of couples undergoing in vitro fertilization. Arch Environ Contam Toxicol 2002; 43: 121-126

Yu AC, Hertz E, Hertz L. Alterations in uptake and release rates for GABA, glutamate, and glutamine during biochemical maturation of highly purified cultures of cerebral cortical neurons, a GABAergic preparation. J Neurochem. 1984; 42: 951-60.

\section{Legends}

Figure 1.- Effects of prolonged exposure to dieldrin on glutamatergic neurotransmission. Primary cultures of cortical neurons were continuously exposed to $60 \mathrm{nM}$ dieldrin for 6 days in vitro (DIV) from the second day in vitro. Top panel: glutamate activity on both ionotropic (NMDA as agonist) and metabotropic (DHPG as group $1 \mathrm{mGluR}$ agonist) receptors, as measured by the increase in intracellular calcium, was reduced by dieldrin. Bottom panel: dieldrin induced the internalization of NMDA receptor subunits and the down-regulation of the mGluR5, in agreement with the loss of function of NMDA and group-1 
metabotropic glutamate receptors. Redrawn from Briz et al., 2010, with permission.

Figure 2.- Effects of prolonged exposure to methylmercury $(\mathrm{MeHg})$ on primary cultures of cerebellar granule cells. Top panel, left: Cultured cells were continuously exposed to methylmercury for $6(\mathbf{-}), 11(\mathbf{A})$ or $16(\boldsymbol{\nabla})$ days in vitro (DIV) and cell viability was determined by means of the MTT assay. LC50 values were in the range $200-500 \mathrm{nmol} / \mathrm{L}$. Top panel, right: Cultured cells were continuously exposed to $300 \mathrm{nM}$ methylmercury for 4 to 8 days in vitro. Methylmercury produced significant cell death after at least 5 days of in vitro exposure. Bottom panel: Glutathion peroxidase activity (left) and lipid peroxidation (right) in cells exposed to $300 \mathrm{nM}$ methylmercury (MeHg) in the absence and in the presence of antioxidants (ascorbic acid, AA; trolox, TX; and probucol, PB) for 5 days in vitro. Redrawn from Farina et al., 2009 and Vendrell et al., 2010, with permission. 
Figure 1
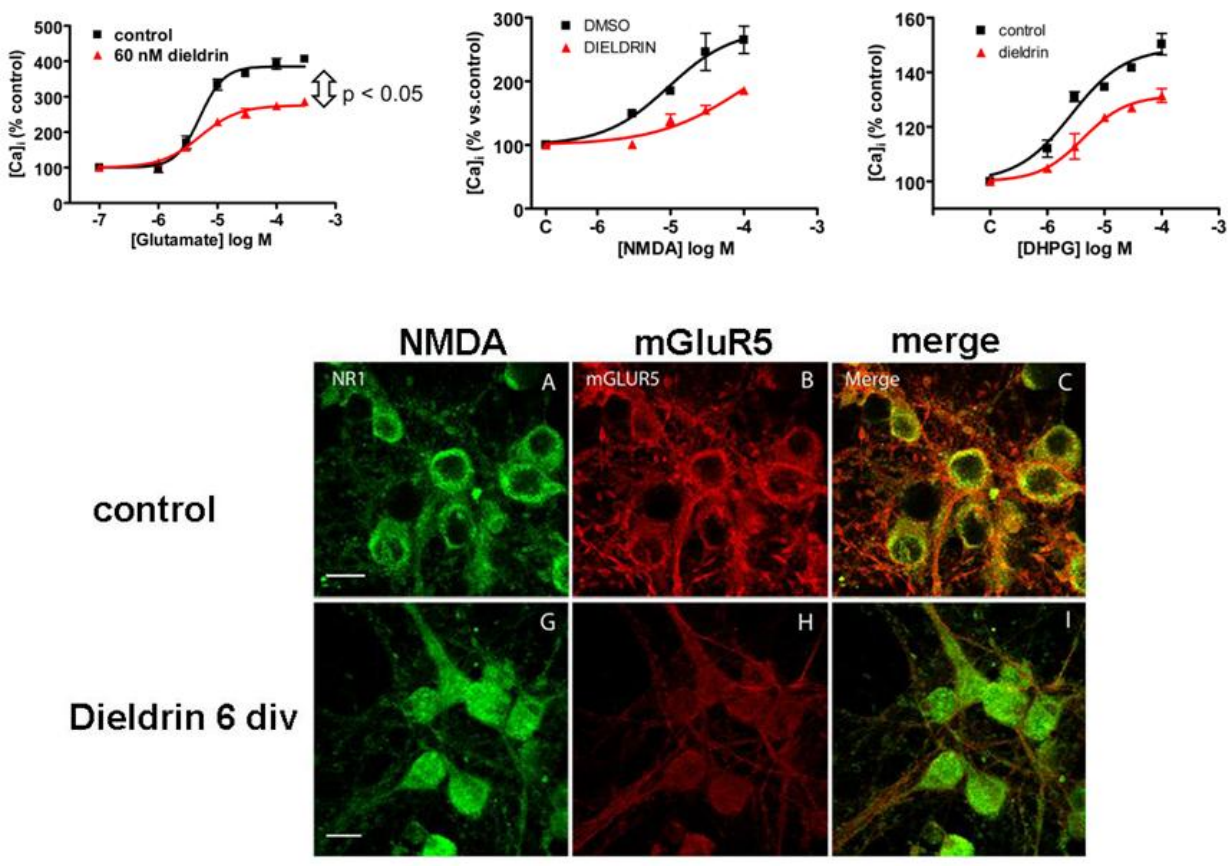
Figure 2
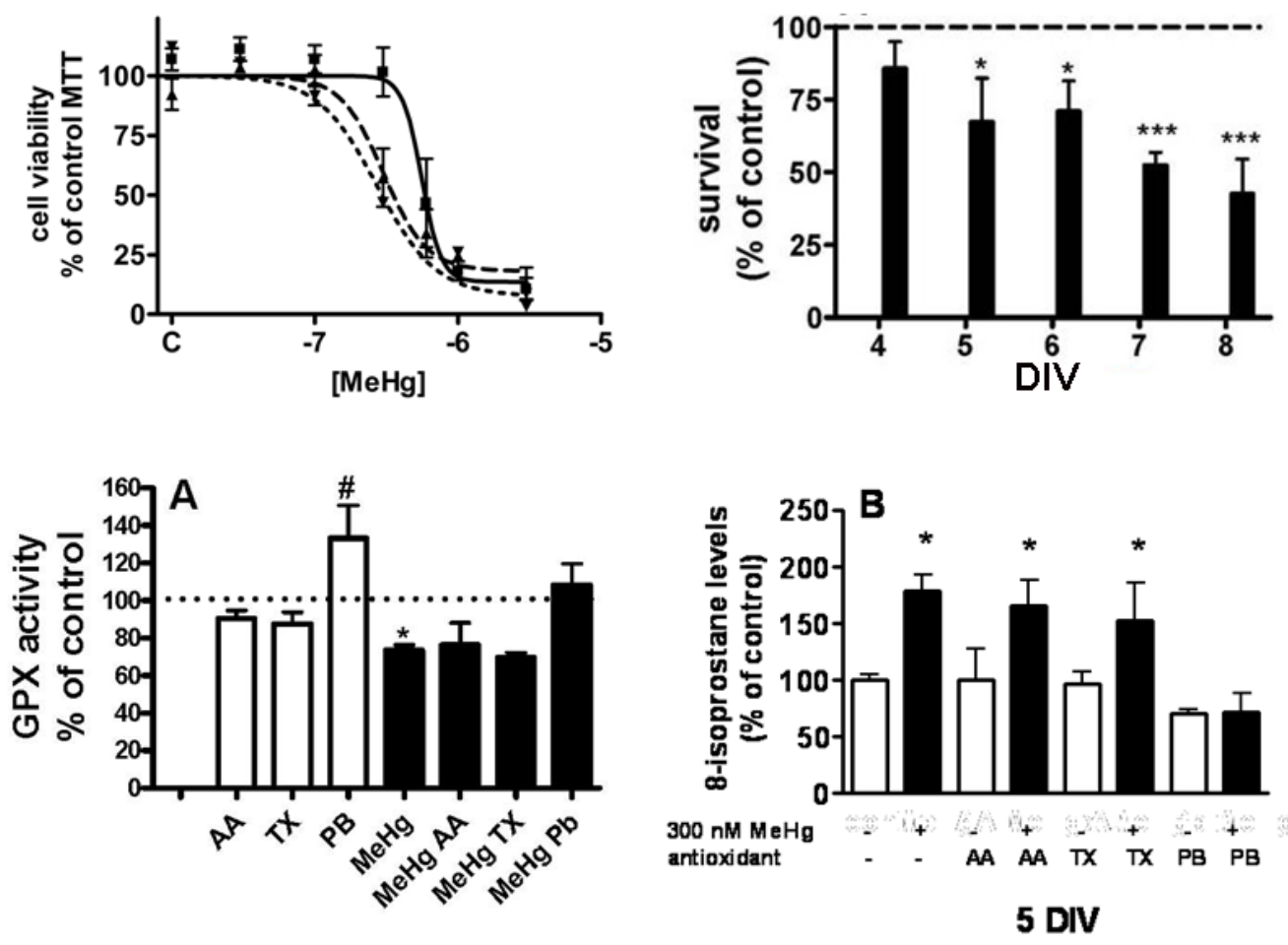